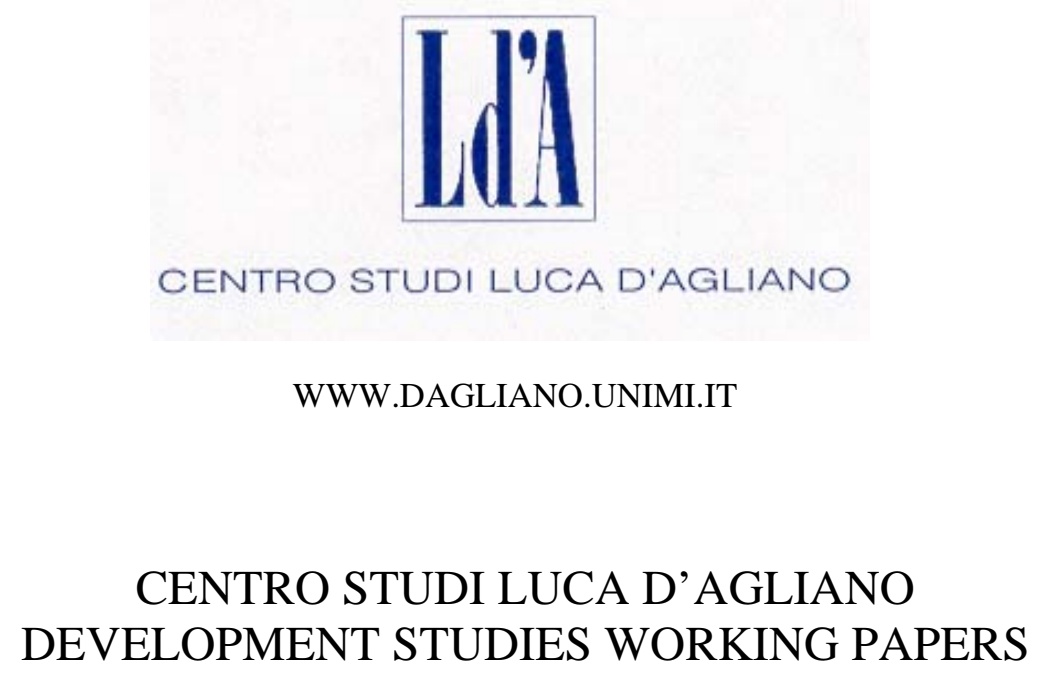

N. 227

May 2007

\title{
The Evolution of the World Trade and the Italian 'Anomaly': A New Look
}

\author{
Michele Di Maio* \\ Federico Tamagni**
}

* University of Macerata

** Sant'Anna School of Advanced Studies 


\title{
The evolution of world trade and the Italian 'anomaly':
}

\section{a new look}

\author{
Michele Di Maio* \\ University of Macerata
}

\author{
Federico Tamagni \\ Sant'Anna School of Advanced Studies
}

February 26, 2007

\begin{abstract}
This work provides an empirical assessment of the 'sophistication' of the Italian international specialization pattern and of its evolution during the period 1980-2000. In particular we discuss the Italian 'anomaly', i.e. the evidence that Italy displays a specialization pattern more similar to the one of emerging economies than to the one of countries of comparable level of per-capita income. We show that combining the information coming from a new index measuring the income/productivity content of traded goods, i.e. the PRODY index recently proposed in Hausmann et al. (2005), with the index of Revealed Comparative Advantages (RCA) can shed light on the Italian anomaly.

We begin providing a detailed picture of the theoretical and empirical characteristics of the PRODY index. In particular we calculate the index for 1980, 1990 and 2000 mapping its dynamics through that period. Then we describe the characteristic and evolution of the Italian RCA using both parametric and non parametric techniques finding that the Italian pattern of specialization is particularly persistent. Finally, we describe the co-evolution of the PRODY and of the RCA indexes. Our analysis shows that in the last two decades, the world trade has been rapidly changing with Italy becoming increasingly more competitive and specialized in products that are characterized by decreasing income/productivity levels. Thus, while the Italian 'anomaly' was not a problem in the past, it may have become an obstacle to future growth.
\end{abstract}

\section{JEL codes: C14, F14}

Keywords: Specialization pattern, RCA, PRODY index, Italian 'anomaly'

\footnotetext{
*Corresponding author: Michele Di Maio, DIEF, Universitá di Macerata, via Crescimbeni 20, 62100 Macerata. Tel+39-0733-258235. E-mail: m.dimaio@unimc.it. We are very grateful to Luca De Benedictis, Giovanni Dosi and Lucia Tajoli for many insightful discussions. We also thank participants to the $11^{\text {th }}$ International Schumpeter Society Conference, Nice-Sophia Antipolis, June, 21-24, 2006, to the 47a Riunione della Societá Italiana degli Economisti, Verona, 27-28 October, 2006, and to the CNR Seminar on International Trade and Development, Turin, 22-23 February, 2007 for very useful comments. Usual disclaimers apply.
} 


\section{Introduction}

There is large consensus among economists and policy makers on the fact that economic growth is to an important extent determined by the country's external performance. A number of theoretical models have also shown that the strength of this link heavily depends on the 'sophistication' of the country specialization pattern (Kaldor (1966); Thirwall (1979); Pasinetti (1981); Dosi et alt. (1990); Grossman and Helpman (1991)).

The debate on the last decade(s) of Italian economic peformance has repeatedly made a large use of this theoretical apparatus to support the view that one major factor of difficulty for the country laid in a mis-directed pattern of specialization. 1 Indeed, it is a very well known fact that Italy is characterized by a peculiar trade 'anomaly' (Onida (1999)), consisting in the fact that 1) Italy is highly specialized in low-skilled labor intensive sectors, implying that the Italian specialization pattern is more similar to the one of emerging economies than to the one of countries of comparable level of per-capita income; and 2) the specialization pattern is highly persistent (Epifani (1999); Monti (2005); De Benedictis (2006)).

While theoretically well founded, the view that what matters for economic growth is the 'sophistication' of the specialization pattern is very difficult to test empirically and very few studies have attempted to do that. For instance, Dalum et al. (1999) shows that the characteristics of the specialization pattern are important to explain growth differentials, but the results are very sensitive to the classification of the sectors in the different 'technological' categories that the authors consider. Feenstra and Rose (2000) find a strong relation between what they call "advanced export structure", and high productivity levels and fast growth rates. These results strongly indicate that it is not openness per se that is growth enhancing, but, rather, the type and characteristics of the sectors wherein the country is specialized that matter.

An important contribution to this line of research comes from the paper by Hausmann et al. (2005) where a new quantitative measure of 'sophistication' of exports is presented. Specifically, they introduce an index which returns, for each traded sector or product, a weighted average of the per-capita income levels of the countries which are exporting in that particular sector or product, whence the name PRODY. In this way, all the sectors or products are ranked in terms of their 'productivity content', and, building on that, they construct a quantitative measure of the 'sophistication' of the overall specialization pattern of each country, called EXPY, which is shown to be a good predictor of subsequent growth.

Drawing on these recent evidence, in this paper we presume that the pattern of specialization, i.e. the specific products which a country exports, do matter for growth. The interesting aspect of the Italian anomaly lays exactly in the fact that, notwithstanding its apparently misdirected specialization pattern, for a long period Italy has recorded good growth rates. Why isn't this happening anymore?

Our paper is an attempt to answer this question. To do that, we structure the analysis into three steps. We begin by using the PRODY index as a mean to offer a detailed picture

\footnotetext{
${ }^{1}$ For an opposite view see Faini (2004).
} 
about how world trade has evolved in the last decades. Extending the work by Hausmann et al. (2005), we analytically derive some dynamic properties of the index and compute its value for three years, 1980, 1990, and 2000, over a sample of 90 countries and 777 traded products. The evidence we found seems to suggest that the entry of many low and mid developed countries into international trade has played a major role in producing the observed change occurred in the sectoral ranking of export 'sophistication' over the last twenty years. Then, as a second step, we turn to Italy and describe the characteristics and the evolution of its specialization pattern between 1980 and 2000. Though richer than previous ones in terms a finer level of sectoral disaggregation we are able to apply, the analyses confirm much of the existing evidence on Italy: persistently increasing and polarized specialization characterizes the period under consideration. Finally, in the third part of the paper, the findings about the evolution of the PRODY index are confronted with the evidence on Italy to attempt a quantitative assessment about the evolution of the 'sophistication' of the Italian specialization pattern. The result seems to suggest a precise answer to our question. Indeed, whereas during the last two decades the emergence of new competitors and a vast world-wide redistribution of production have taken place at the world level, both causing significant changes in the relative gains associated to exporting different products, Italy has remained stuck to its original positioning. In addition, a strong decline is observed in the values of the PRODY index for many of the sectors where Italy has persistently been, and still is, highly specialized. The evolution of world trade scenario seems therefore the very reason why the Italian anomaly, though it has not been a problem for a long time, yet contributing to a good performance in terms of growth, it is now starting to become a point of weakness and the time has come to worry about it.

The paper is organized as follows. In section 2 we present the PRODY index and we discuss some theoretical aspects concerning its dynamic properties. In section 3 we present a set of empirical exercises exploring the evolution of the index in the period $1980-2000$. The analysis of the evolution of the Italian RCA in the same period period is then presented in section 4, while Section 5 combines the two sets of empirical results to evaluate the 'sophistication' of the Italian specialization pattern. Section 6 concludes and suggests some lines for further research.

\section{Measuring the productivity content of traded goods: the PRODY index}

In this section we introduce the PRODY index and we analytically derive some of its dynamic properties. Recently proposed in Hausmann et al. (2005), the index ranks each traded sector in terms of the income/productivity level of the countries that export in that specific sector. Formally, it is defined as

$$
\operatorname{PRODY}_{l}=\sum_{i=1}^{N} s_{i, l} \cdot y_{i}
$$


where $y_{i}$ is real GDP per capita of the $i$-th $(i=1,2, \ldots, N)$ country exporting in sector $l$, and the weight

$$
s_{i, l}=\frac{R C A_{i, l}}{\sum_{i} R C A_{i, l}}
$$

normalizes the Balassa index of Revealed Comparative Advantage (RCA) of the $i$-th country with respect to those of all the countries exporting in the same sector.2 Given the definition, sectors with high values of $P R O D Y$ are, by construction, those where high income countries play a major role with respect to the other participants in world exports in that sector. In other words, the index measures the degree of 'sophistication' of sectors (goods) with the level of development of the countries producing and exporting in that sector, under the implicit (and reasonable) assumption that the presence of high income/high wages countries is stronger where comparative advantage are determined by factors other than labor cost, such as know-how, technology or intrinsic quality. To the extent that such factors set the stage for non-perfectly competitive environments, high PRODY sectors end up being growth enhancing as they should ensure high profit margins to the countries particularly well positioned in their production and trade. $3^{3}$

While Hausmann et al. (2005) and Rodrik (2006) compute only a single value of the PRODY indices, providing a three-year average for the period 1999-2001, one of the contribution of our paper is to provide an analysis of its dynamics over time. The idea is that, given the aforementioned association between 'sophistication' of traded goods and income level of the countries exporting them established by the sectoral PRODY indeces, significant changes in their values and their ranking over time might reveal that significant mobility and transformations have taken place in the international division of labour which, in turn, might have modified the potential contribution of trading different goods to overall economic growth.

Interpreting the dynamics of the index requires to understand how the index itself responds to changes in its two components, that is the extent of specialization (i.e. the RCA index) and per capita income of the countries exporting each particular product, and to changes in the number of countries involved, as determined by the processes of entry and exit in sectoral export markets over time. The following propositions summarise what one can say about these effects. First of all one has

Proposition 1 The effect of a change in country k's extent of specialization in sector $l$ on the PRODY index of the same sector intrinsically depends on its effect on the other's country extent

\footnotetext{
${ }^{2}$ Recall that the Balassa-RCA index is defined as:
}

$$
R C A_{l i t}=\frac{X_{l i t} / X_{i t}}{X_{l w} / X_{w}}
$$

where $X$ denotes the value of exports, $l$ denotes the sector, $i$ the country, $t$ the year considered, while $w$ indicates the world economy. The index gives a measure of how important (in terms of value) is sector $l$ in country $i$ 's exports bundle with respect to the importance sector $l$ has in total world exports. If the index is larger (less) than 1 , country $i$ is said to be specialized (de-specialized) in sector $l$.

${ }^{3}$ On this interpretation see also Lall et al. (2006). 
of specialization in that sector. Indeed

$$
\frac{\partial P R O D Y_{l}}{\partial R C A_{k, l}}=\sum_{i} y_{i} \cdot\left(\frac{\frac{\partial R C A_{i, l}}{\partial R C A_{k, l}}\left(\sum_{j} R C A_{j, l}\right)-\left(\sum_{j} \frac{\partial R C A_{j, l}}{\partial R C A_{k, l}}\right) R C A_{i, l}}{\left(\sum_{j} R C A_{j, l}\right)^{2}}\right)
$$

Second, concerning the effect of changes in income levels of already exporting countries, the following holds.

Proposition 2 Ceteris paribus, an increase in the income of a country exporting in sector $l$ increases the value of the PRODY index of the same sector proportionally to its share in world exports of that product. Indeed

$$
\frac{\partial P R O D Y_{l}}{\partial y_{k}}=s_{k, l}
$$

which is always positive.

Finally, the way in which the entry of a new exporter in a particular sector affect the index crucially depends on the relative income levels of the countries involved. The following holds

Proposition 3 The probability of observing an inter-temporal increase of the index is higher the more developed the entrant country is, and the less developed the already exporting countries are. Indeed, if $A$ and $B$ are already present in world trade of good $l$, while $C$ is the new exporter, then

$$
\Delta P R O D Y_{l}>0 \Leftrightarrow R C A_{C, l}>\underbrace{-\Delta R C A_{A, l}}_{>0} \cdot \frac{y_{A}}{y_{C}} \underbrace{-\Delta R C A_{B, l}}_{>0} \cdot \frac{y_{B}}{y_{C}}
$$

Proof. See Appendix

This latter result is particularly important because it will be useful when, in section 3, we will try to offer an explanation to what observed in the data. Proposition 3 also makes clear that, to avoid mis-measurement of the PRODY index, it is essential to gather data on the biggest possible number of countries and to keep all of them in the sample for the whole period considered. In the Appendix we describe the choices made in order to cope with these issues.

\section{Evidence on the dynamics of PRODY}

We begin analysing how the sectoral PRODY indexes evolved during the sample time span. Our trade data come from the UN Commodity Trade Statistics Database (COMTRADE). For each country present in the dataset it contains detailed yearly figures on the value of exports in 777 sectors (SITC Rev.2 classification, 4-digit) and aggregate data on the economy wide level of exports. We used Penn World Tables 6.5 for data on per-capita income, measured in 1996 US dollars, PPP adjusted.

Some descriptive statistics for the three reference years 1980, 1990 and 2000 are shown in Table 1. Three features seems to emerge. First, the values of the indices varies greatly within 
each year, from numbers in hundreds to tens of thousands of 1996 US dollars per capita. Second, it seems that the importance of sectors characterized by low values has increased over time. Indeed, the distribution of the index shifts toward the left: the 1-st quartile and the median both decrease steadily, whereas the mean and the 3-rd quartile, after an increase between 1980 and 1990, in 2000 end up below their initial values. Third, the standard deviation (SD) also increases, suggesting that the support of values has widened, and the distance between goods with high or low income/productivity content too.

Table 1: PRODY index, descriptive statistics

\begin{tabular}{lrrr}
\hline \hline & $\mathbf{1 9 8 0}$ & $\mathbf{1 9 9 0}$ & $\mathbf{2 0 0 0}$ \\
\hline \hline Min. & 200 & 92 & 123 \\
1st Qu. & 6567 & 6304 & 4568 \\
Median & 10051 & 9882 & 8172 \\
Mean & 9582 & 9904 & 8591 \\
3rd Qu. & 12860 & 13441 & 11740 \\
Max. & 20740 & 20571 & 24420 \\
SD & 4177 & 4562 & 4851 \\
\hline \hline
\end{tabular}

Values in 1996 PPP-adjusted US dollars per capita.

We will return on these features below, when we will come back to the analysis of the changes occurred in the overall distribution. Before doing that, we look at average persistence over time.

In Table 2 we report the Spearman rank correlations estimated for the 10 and 20 years transition of the indexes. The values, all above 0.5 and all significant, tell us that a positive relationship is in place across time, though decreasing between the 80's and the 90's.

Table 2: PRODY index, rank correlations

\begin{tabular}{ll}
\hline \hline $1980-1990$ & $0.751^{*}$ \\
$1990-2000$ & $0.712^{*}$ \\
$1980-2000$ & $0.660^{*}$ \\
\hline \hline
\end{tabular}

$\left({ }^{*}\right)$ Coefficients significant at $5 \%$ confidence level

We further explore the issue in Table 3 where we show the result of a so-called Galtonian regression, which is a standard way to get a synthetic measure of cross-section inter-temporal persistence with respect to an initial period. The estimated equation is:

$$
P R O D Y_{l, t+n}=\alpha+\beta \cdot P R O D Y_{l, t}+\epsilon_{t}
$$

where $l$ is the sector index and $t$ is the year, where the estimated coefficient $\beta$, captures what 
happens to the conditional average of the cross-sectoral distribution. The previous picture is substantially confirmed: there is a positive relationship over time, but weaker in the '90s than in the '80s. Moreover, the extent of such relationship is decreasing as we move from the 10 years to the 20 years transition.

Table 3: PRODY index, Galtonian regression

\begin{tabular}{lccc}
\hline \hline & $\mathbf{1 9 8 0 - 1 9 9 0}$ & $\mathbf{1 9 9 0 - 2 0 0 0}$ & $\mathbf{1 9 8 0 - 2 0 0 0}$ \\
\hline \hline Intercept & $2215.29^{*}$ & $1288.79^{*}$ & $1386.96^{*}$ \\
& $(3.3 \mathrm{e}+02)$ & $(2.7 \mathrm{e}+02)$ & $(3.1 \mathrm{e}+02)$ \\
$\beta_{P R O D Y}$ & $0.802^{*}$ & $0.737^{*}$ & $0.752^{*}$ \\
& $(0.032)$ & $(0.030)$ & $(0.034)$ \\
\hline \hline N.Obs. & 777 & 777 & 777 \\
$R_{\text {adj }}^{2}$ & 0.539 & 0.480 & 0.418 \\
$\mathrm{~F}$ & $909.1^{*}$ & $717.8^{*}$ & $559.3^{*}$ \\
\hline \hline
\end{tabular}

(*) Significant at $1 \%$ confidence level.

\subsection{Distributional dynamics of the PRODY index}

The limits of the previous analyses are known. Among the many, Quah (1996) and Durlaf and Quah (1999) discuss the issues related to the so called Galton-fallacy problem in the context of crosscountry growth regressions, and clarify the merits of focusing on a characterization of the entire distribution of a variable, and of its evolution over time. This is the route we take.

Figure 1 shows the estimated kernel densities of the values (in logs) taken by the PRODY indexes in the 777 sectors we have in our sample, for 1980, 1990 and 20004. Two features seems to characterize the time evolution of the distribution. First, concerning the central part of the distributions, one can see that the densities estimated in the three reference years have similar shape, with a peak around 9.5, and long left tail, but the one estimated for 1980 is much more concentrated around the modal value. During the two subsequent decades the support spanned becomes wider and the height of the peak falls considerably, suggesting that a relevant part of the probability mass is shifting to the left: as time passes, the values of the indeces become more heterogeneous, and most of the sectors seems to experience a reduction in the degree of sophistication. Second, focussing on the behavior in the tails, one can conclude that the left shift observed in the most relevant part of the probability mass is accompained by a process of polarisation. Indeed, both the lower and the upper tail are heavier in 2000 than they were

\footnotetext{
${ }^{4}$ All the exercises performed in this work, when not else specified, used gbutils, a package of programs for parametric and non-parametric analysis which is distributed under the General Public License, and freely available at www.sssup.it/ bottazzi/software. Kernel density estimations, in this section and in the followings are performed using Epanenchnikov kernel and setting the kernel bandwidth according to the "optimal routine" suggested in Silverman (1986).
} 
Figure 1: PRODY index, kernel densities in 1980, 1990 and 2000.

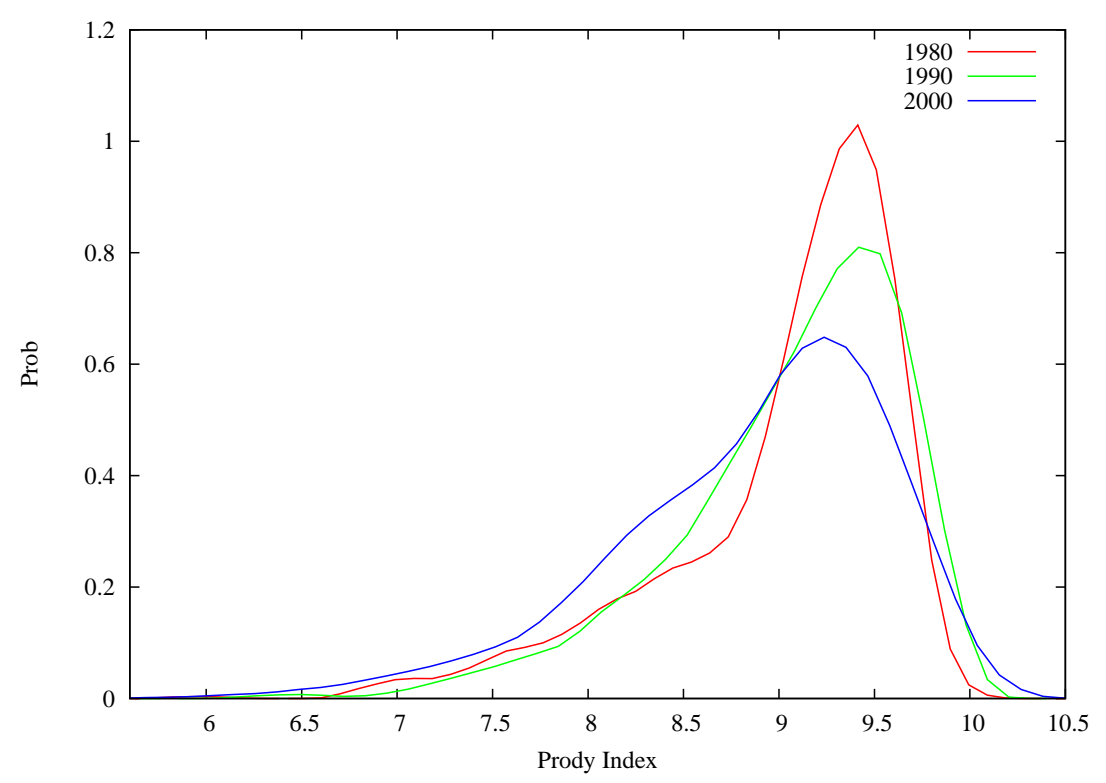

in 1980, meaning that the proportion of sectors displaying the lowest and biggest value of the index have both increased over time.

The discussion we exposed above concerning the dynamic properties of the PRODY index offer a way to interpret this picture. Proposition 3 suggests that a decrease in the value of $P R O D Y_{l}$ is much more likely to happen when low-medium income countries are entering the scene of world trade in sector $l$. The clearcut swing towards the left observed over time in a big part of the probability mass, and the associated decrease in the index of many sectors, represent two findings quite consistent with this story.

A similar line of reasoning can be followed also in explaining the results about the movements occurring in the upper tail. Read in view of Proposition 3, there are two possible explanations (not mutually exclusive) for the increase taking place in both the values and the weight of this tail. Either the developed countries already active in these sectors have further reinforced their extent of specialization, or, if any other player entered these markets, they should have been high income countries.

Overall, the analyses of the distribution of the PRODY index performed up to now seems to suggest an evolution of world export characterized by low or medium developed countries entering into the sectors characterized by lower technological content, while developed countries being comparatively more active in sectors with higher values of the index. To corroborate this interpretation it would be essential to understand how stable it has been the ranking of the sectors in terms of their PRODY index. Indeed, one would like to understand whether the best (worst) performing sectors in 1980 are still the best (worst) ones in 1990 and 2000 or not. Being this the case, this would reinforce the idea that a dichotomy has emerged between sectors where it has been essential to be present, because they stably warranted a closer link with income/productivity frontier, and other sectors which, in the course of the last 20 years, have always become less and less important. On the contrary, observing a clear change in the 
Figure 2: PRODY index, contour plots of the joint kernel density between 1980 and 1990 (left) and between 1980 and 2000 (right).
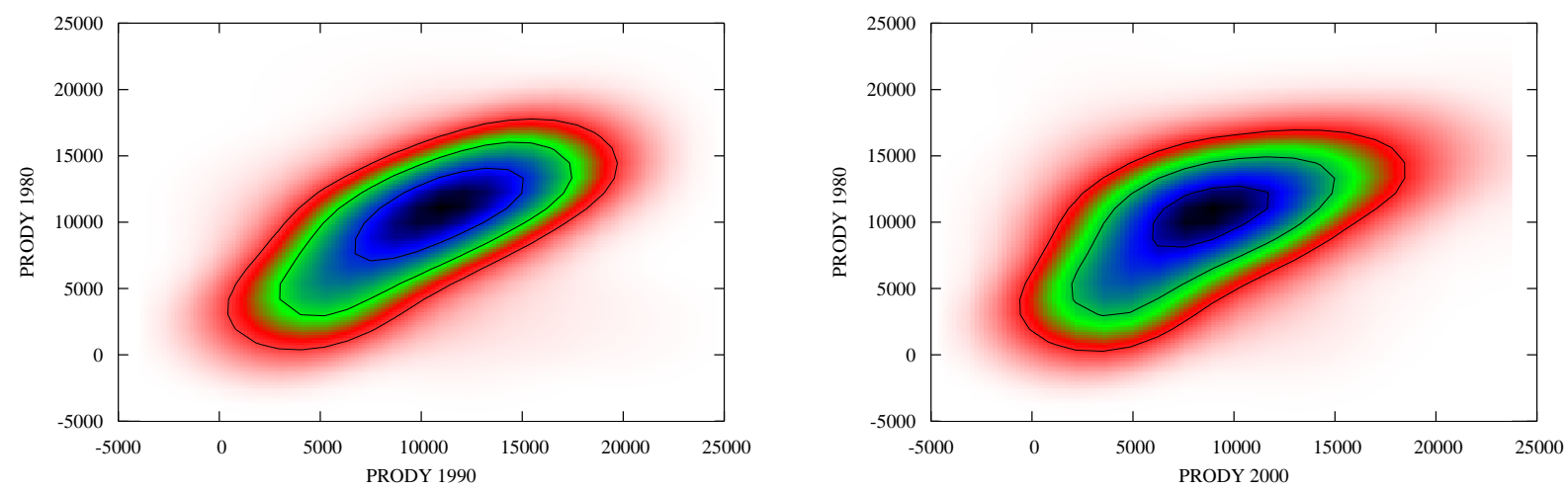

ranking of the PRODY indexes would suggest that international trade patterns continuously evolves. In this case, to understnd how specialization changes over time is crucial, as an high potential 'productivity' associated with sector $l$ in the 80's might well have turned into a very low level in 2000. To tackle these issues in more detail we look at the 10 and 20 years bivariate kernel density of the PRODY indexes. 5

In the left panel of Figure 2 we report the contour plots of the joint density of the PRODY index between 1980 and 1990. The values of the index in 1990 are on the $x$-axis, so that the points lying on an imaginary positively sloped bisector represent sectors for which the index remained unchanged, while points above (below) it identify sectors where the index is estimated to have decreased (increased).6 A first look to the plot reveals that the most part of the distribution lies only slightly above the positively sloped bisector: consistently with Figure 1, during the 80 's, there has been a slight tendency toward an increase in the importance of the sectors ranked among the low or medium performing in terms of income/productivity content. But actually we can add something more. Indeed, the presence of a positive relationship between the distributions in the two years suggests a considerable degree of stability in the ranking of the sectors: those with the lowest (highest) PRODY in 1980 are likely to display a lowest (highest) value of the index also in 1990. Something different emerges from the right panel of the same Figure 2, where we perform the same exercise, but focusing on a longer time horizon, that is between 1980 and 2000. Though the overall picture speaks again in favor of stability, we can note that there is a shift towards the up-left corner, and the central part of the distribution lies now above the bisector, at least more than it was in the period 1980-1990. Consistently with Figure 1, many sectors experienced a decrease in the index, and a mild polarization emerges, where sectors with highest values PRODY in 1980 have an even higher one in 2000.

\footnotetext{
${ }^{5}$ See also the Appendix where we describe in greater detail the dynamics of the best performing 20 sectors, following the evolution of their PRODY indeces from the beginning to the end of the sample period.

${ }^{6}$ Negative values are the mere result of the smoothing operated by kernel estimates.
} 
Table 4: $R C A$ of Italy, rank correlations

\begin{tabular}{ll}
\hline \hline $1980-1990$ & 0.794 \\
$1990-2000$ & 0.820 \\
$1980-2000$ & 0.754 \\
\hline \hline
\end{tabular}

Coefficients significant at $5 \%$ confidence level

Overall, our analysis of the characteristics of the PRODY index suggests that between 1980 and 2000 something has changed in the characterstics of world trade 'sophistication'. The evolution we have illustrated and measured in this section indicates that what was 'good' exporting in 1980 might not necessarily coincide with what was 'good' in 2000. To the extent that the index is effective in capturing the potential contribution to aggregate growth stemming from exporting in each particular sector, the main message we draw is that it should not have been neutral whether one country's international specialization has evolved towards one direction or another. This suggests that, in order to assess how 'good' the pattern of specialization of a country is, it could be instructive to compare the evolution of the PRODY index with that of the $R C A$. In the following we will apply this idea considering the Italian case.

\section{The evolution of Italian comparative advantages}

We describe the Italian specialization by means of the standard Balassa-RCA index. In parallel with the exercises run in the analysis of $P R O D Y$, we begin looking at the average picture computing rank correlations among 10 and 20 years transition, reported in Table 4. As commonly observed when looking at most developed countries, the correlation values are fairly high, suggesting that, if changes in the specialization pattern have taken place, they should have been of minor nature. Notably, the strength of the relationship is increasing over time, and this seems a peculiarity of the Italian case. $]^{7}$

This result is also confirmed by running a Galtonian regression of the form

$$
R C A_{l, t+n}=\alpha+\beta \cdot R C A_{l t}+\epsilon_{t}
$$

where, as usual, $l$ stands for the sector, while $t$ and $t+n$ indicate the initial and final year, respectively.

The results reported in Table 5 confirm a quite high level of persistence in the pattern of specialization. The value of the coefficients $\beta$ are all positive, significant and rather high. 8 Interestingly, the degree of persistence has increased over time. Indeed, although a process of regression to the mean is working over the entire period at stake, this seems occurring faster in the first ten years $(\beta \simeq 0.62)$ than in the second decade $(\beta \simeq 0.92)$.

\footnotetext{
${ }^{7}$ For a cross-country comparison see De Benedictis and Tamberi (2004).

${ }^{8}$ Robust standard errors are obtained via Huber (1967) "sandwich" estimator of the variance/covariance matrix.
} 
Table 5: $R C A$ of Italy, Galtonian regression

\begin{tabular}{lccc}
\hline \hline & $\mathbf{1 9 8 0 - 1 9 9 0}$ & $\mathbf{1 9 9 0 - 2 0 0 0}$ & $\mathbf{1 9 8 0 - 2 0 0 0}$ \\
\hline \hline Intercept & $0.258^{*}$ & $0.211^{*}$ & $0.386^{*}$ \\
& $((0.0373)$ & $(0.0429)$ & $(0.0520)$ \\
$\beta_{R C A}$ & $0.623^{*}$ & $0.992^{*}$ & $0.682^{*}$ \\
& $(0.01804)$ & $(0.02558)$ & $(0.02512)$ \\
\hline \hline N.Obs. & 777 & 777 & 777 \\
$R_{a d j}^{2}$ & 0.605 & 0.659 & 0.487 \\
$\mathrm{~F}$ & $1193^{*}$ & $1506^{*}$ & $738^{*}$ \\
\hline \hline
\end{tabular}

(*) Significant at $1 \%$ confidence level

Figure 3: $R C A$ of Italy, kernel densities over time

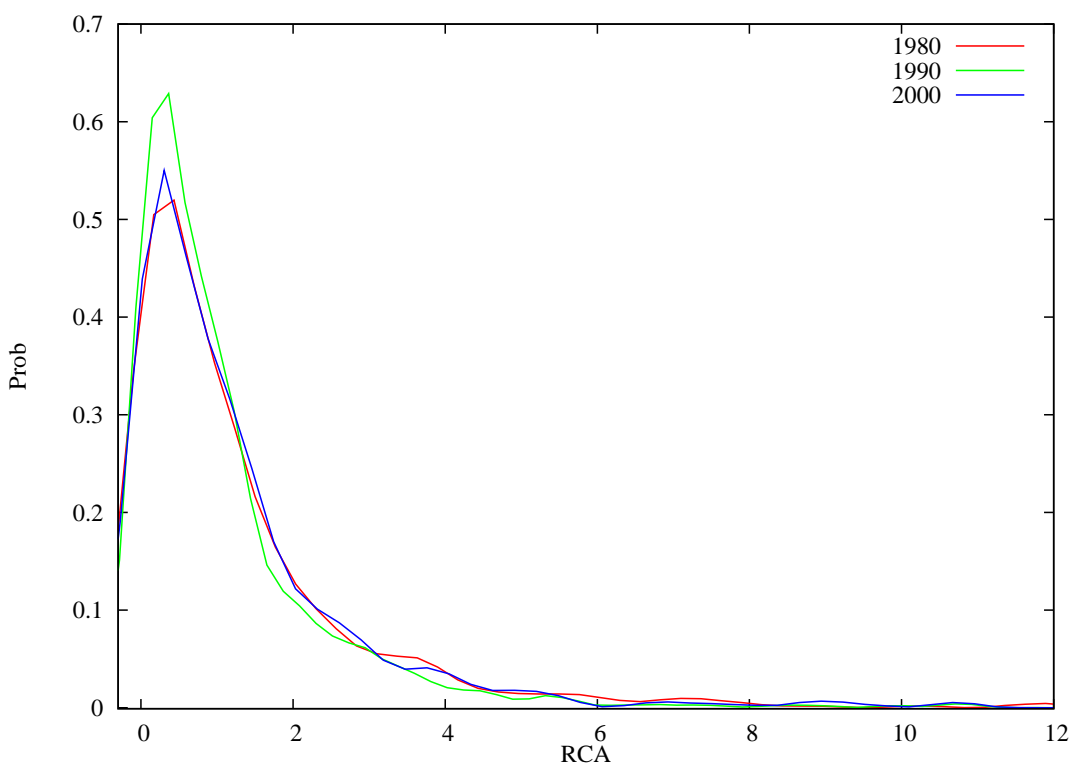

\subsection{Distributional dynamics of the $R C A$ index}

We now move to describe the overall distributional dynamics of the Italian specialization pattern.9 We first estimate, for each year in the period 1980-2000, the kernel density of the sectoral $R C A$ indexes. Since we observed a high degree of stability in the shape of the yearly distributions, in Figure 3 we report and comment results only for the three reference years 1980, 1990 and 2000 .

Comparing the three distributions, it seems that not much has happened during the period under consideration. Motivated by previous contributions focussing on the properties of Italian RCA, we also checked for the presence of bi-modality, which is confirmed by a formal test

\footnotetext{
${ }^{9}$ Other examples of application of entire distribution or intra-distribution analysis to trade empirics are the works by Proudman and Redding (2000), Brasili et al. (2000), De Benedictis and Tamberi (2004), and De Benedictis (2006).
} 
Table 6: RCA of Italy: bimodality test

\begin{tabular}{ccccc}
\hline \hline & $\mathrm{h}(1)$ & $\mathrm{p}$-value & $\mathrm{h}(2)$ & $\mathrm{p}$-value \\
\hline $\mathbf{1 9 8 0}$ & 1.395 & 0.351 & 0.546 & 0.665 \\
$\mathbf{1 9 9 0}$ & 0.991 & 0.256 & 0.609 & 0.271 \\
$\mathbf{2 0 0 0}$ & 1.379 & 0.073 & 0.917 & 0.167 \\
\hline \hline
\end{tabular}

Null Hypothesis: $\mathrm{nr}$. of modes $=1(h(1))$ and $\mathrm{nr}$. of modes $=2(\mathrm{~h}(2))$

Figure 4: $R C A$ of Italy, contour plots of the joint kernel density between 1980 and 1990 (left) and between 1980 and 2000 (right).
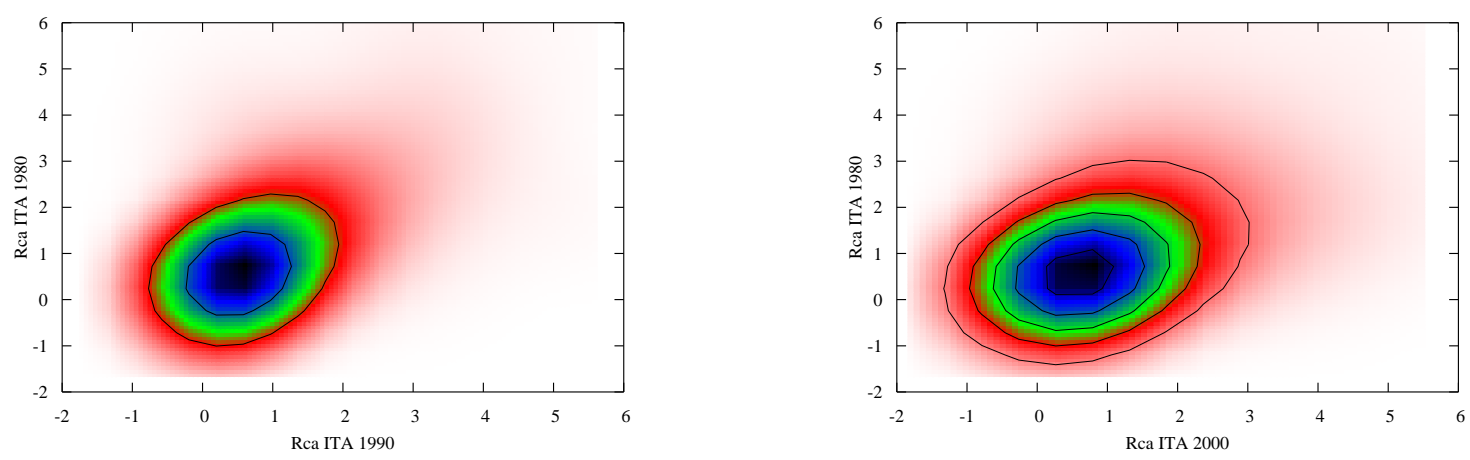

(Silverman 1981, 1983) reported in Table 6. The presence of bi-modality indicates that the specialization pattern of Italy is characterized by the presence of both a large number of sectors in which Italy has very low RCA and of a large(r) number of sectors in which Italy is highly specialized.

To gain further insights, we look at the 10 and 20 years transition dynamics of the entire distribution of $R C A$. Figure 4 shows, in the left panel, the contour plots of the estimated joint kernel density of the RCA index between 1980 and 1990.10 A first look to the plot confirms that not much has happened during that decade and, more importantly, that the ranking of the sectors displays a considerable degree of stability: sectors with high (low) RCA in 1980 are likely to display a high (low) RCA in 1990. However, something more emerges in the right panel of the same figure, where we perform the same exercise, but focusing on a longer time horizon, that is between 1980 and 2000. Though the overall picture speaks again in favor of stability, the wider support spanned suggest a (mild) process of polarization has characterised the evolution of the Italian specialization pattern.

\footnotetext{
${ }^{10}$ Of course negative values are not possible as the RCA index lies in $[0,+\infty]$ by construction: what is observed in the plots is simply the result of the smoothing operated by kernel estimation.
} 


\section{Measuring the 'sophistication' of the Italian specialization pattern}

The description of the characteristics of the Italian specialization pattern given so far, even if accurate and interesting per se, does not provide any information about the 'sophistication' of the Italian international positioning. To fill this gap we perform two exercises exploiting the PRODY index. We first follow Hausmann et al. (2005) in computing an index, called $E X P Y$, that is intended to capture the productivity content associated to the export vector of a country. 11 Then, somehow in the same spirit, we seek to shed some light on whether the Italian sectoral comparative advantages have evolved in line with the changes occurred in the income content of world trade, as captured by the dynamics of the PRODY index.

\subsection{EXPY index for Italy}

The EXPY index is a simple weighted sum of the PRODY indexes computed for all the products exported by country $i$, where the weights are the shares of each particular product in the overall exports of the country. Formally,

$$
E X P Y_{i, t}=\sum_{l}\left(\frac{x_{l, i}}{X_{i}}\right)_{t} \operatorname{PRODY} Y_{l, T}
$$

where $T$ is a reference year in which $P R O D Y_{l}$ is computed, and taken fixed over the sample period, so that the intertemporal changes in EXPY result from how sectoral export shares evolved year by year. Hausmann et al. (2005) takes the three-years $1999-2001$ as reference $T$, and perform a series of exercises exploring the characteristics of the EXPY computed for a panel of several countries during the 90's. Their findings are supportive of the view stressing that the intrinsic 'sophistication' of products, rather than the extent of specialization has important implications for subsequent economic performance. Indeed, they show that the index is highly correlated with GDP and that countries who export goods associated with higher EXPY are those who also grow more rapidly, even after controlling for initial income per head, human capital levels, and time-invariant country characteristics. Thus, it is what an economy exports that matters: rich (poor) countries export products that tend to be exported by other rich (poor) countries and, ceteris paribus, producing and exporting goods that richer countries export represent an effective route to faster growth.

In Figure 5] we compute the value of the index for Italy during the period 1977 - 2004, the entire time window for which trade data are available for this country, and we use the most recent values of the PRODY indexes, that computed for the year 2000, as our benchmark reference point. This means we are mapping the aggregate income/productivity level associated with the Italian export basket when each sector's income/productivity content is held constant over time at values capturing the characteristics of world trade in 2000.

\footnotetext{
${ }^{11}$ This index is similar to the one presented in Michaely (1984) called 'income level of exports'. Lall et al. (2006) have also developed a similar measure that they call the 'sophistication level of exports'.
} 
Figure 5: EXPY index for Italy, 1977-2004

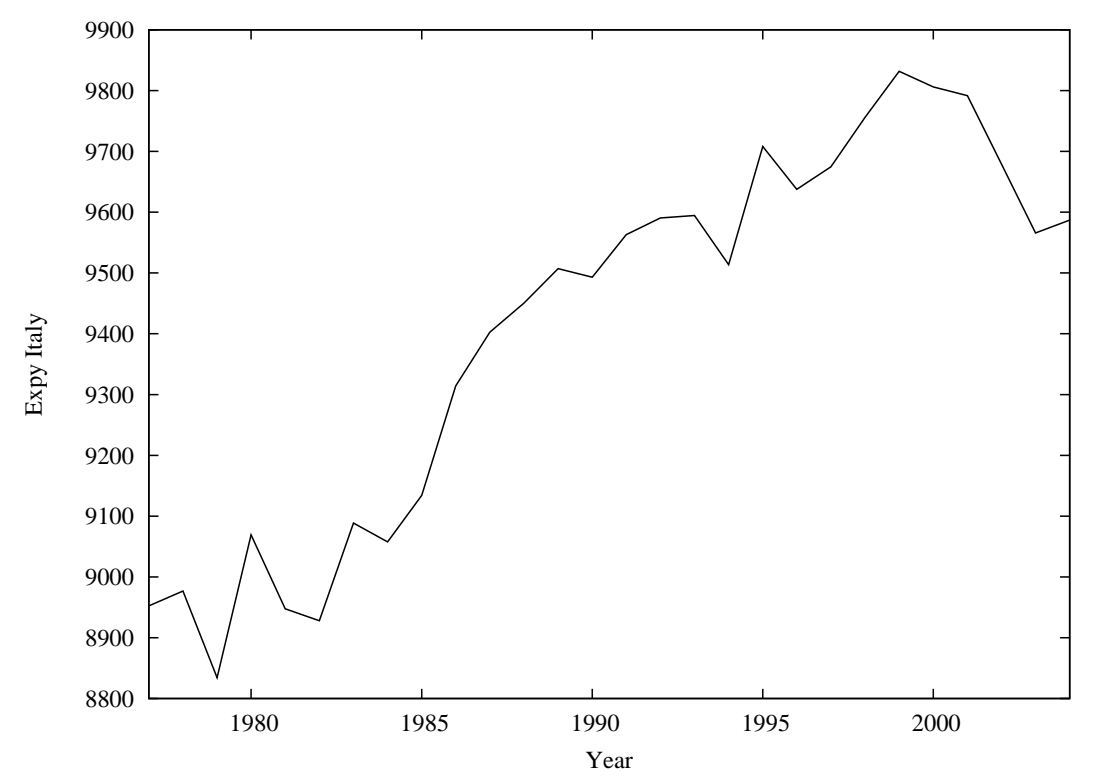

The observed pattern is quite interesting. If one only looks to the overall trend, the conclusion would clearly be that Italy has performed well during the period considered. Indeed, the index has clearly increased over time, from around 8900 USD per capita in 1977, to around 9600 USD per capita in 2004, meaning that a virtuous redistribution of export shares seems to have occurred among the different sectors, with those characterized by higher productivity/income content either increasing their importance or emerging from scratch. Though, some specifications are worth. Indeed, the index steadily increased only until the end of the '90s, while a steep decline has characterized the last 5 years. A break seems occurring around 1999: while the Italian export bundle was moving towards the 'right' direction in the years before, the dynamics in the last part of the period seems suggesting that the pattern of specialization has recently become weaker in terms of implied income/productivity content. In the next section, we will see that looking at the co-evolution between the Italian specialization pattern and the dynamics of the $P R O D Y$ indexes allows to shed some light on the how and why we observe such a break.

\subsection{Dynamics of $P R O D Y$ and the Italian specialization pattern}

With the same goal of providing a synthetic assessment of the 'sophistication' of the Italian specialization pattern, in the following we propose a different way to exploit the information provided by the PRODY indexes. The basic idea is simply to consider the co-evolution of the sectoral Balassa-RCA indexes and the corresponding sectoral PRODY indexes in the three reference years.

In the three panels of Figure 6, we plot, for 1980, 1990 and 2000 respectively, the value of the $R C A$ index relative to each of the 777 sectors in our dataset against the value of the corresponding $P R O D Y$ index, and run a non-parametric estimation of the relationship existing 
Figure 6: Italian RCA vs PRODY, non parametric regression for 1980, 1990 and 2000.
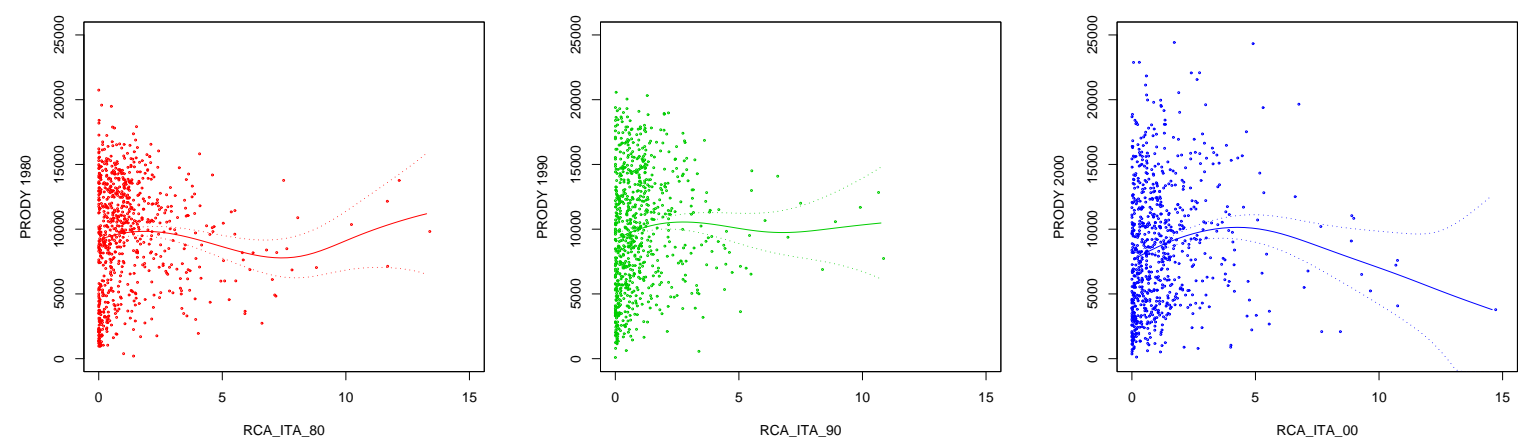

between the two variables. 12

Were Italy mostly specialized in sectors with high $P R O D Y$, the fit would be positively sloped, meaning that sectors where Italy enjoys high (low) $R C A$ are those where $P R O D Y$ is also high (low). Clearly, this would be the preferred situation. Indeed, since high-PRODY products are exported by countries characterized by high per-capita income and thus high wages, it is very likely that these are sectors in which comparative advantages are determined by factors other than labor cost, such as for instance technology 13 Thus, these are likely to be oligopolistic high profit sectors characterized by high value added per unit of product.

Some interesting results emerge from the comparison of the graphs in Figure 6. The relationship between the PRODY index and the $R C A$ index is almost flat (or slightly "inverted-U" shaped) for sectors with low-medium RCA. Things are different for sectors with very high RCA values, say from $R C A>5$. Here a positive relationship is in place in 1980, but then it becomes almost flat in 1990, and turns negative in 2000, suggesting that sectors of strong Italian specialization are those associated with declining 'sophistication'. The overall indication is that the relationship between RCA and PRODY has changed with time, and specifically, it has changed in the direction of a negative relationship between the two 14

To corroborate this conjecture we focus on the 15 sectors in which Italy recorded the highest RCA in 1980, 1990 and 2000, plotted in Figure 7. The above mentioned pattern is confirmed: the cloud of points for these sub-sample of sectors, positively sloped in 1980, ends up negatively sloped in 2000. Moreover, most of the sectors show up in all of the three years, a fact that confirms the high degree of persistence in the top tail of the Italian specialization structure.

Something more can be gained looking at Table 7, where we take the 15 sectors in which Italy was mostly specialized in 2000, and compare the time evolution of their RCA and PRODY indices from 1980 to 2000 . Indeed, we observe a robust pattern suggesting that Italy has maintained

\footnotetext{
${ }^{12}$ The non-linear estimate has been obtained using the $s m$ and kernsmooth library in $R$. We used the standard settings and we also report the variability bands, giving a measure of the statistical significance of the estimate.

${ }^{13}$ As mentioned in Section 2, Lall et al. (2006) propose this interpretation for their index of export sophistication, which is actually much similar to the PRODY and EXPY indexes by Hausmann et al. (2005).

${ }^{14}$ Nonetheless, note that, in all the three graphs, the variability band is larger for high levels of RCA, due to the presence of few observation in that area. Thus, also our interpretation is to be taken with caution.
} 
Figure 7: Top 15 Italian RCA and the associated PRODY in 1980, 1990 and 2000.
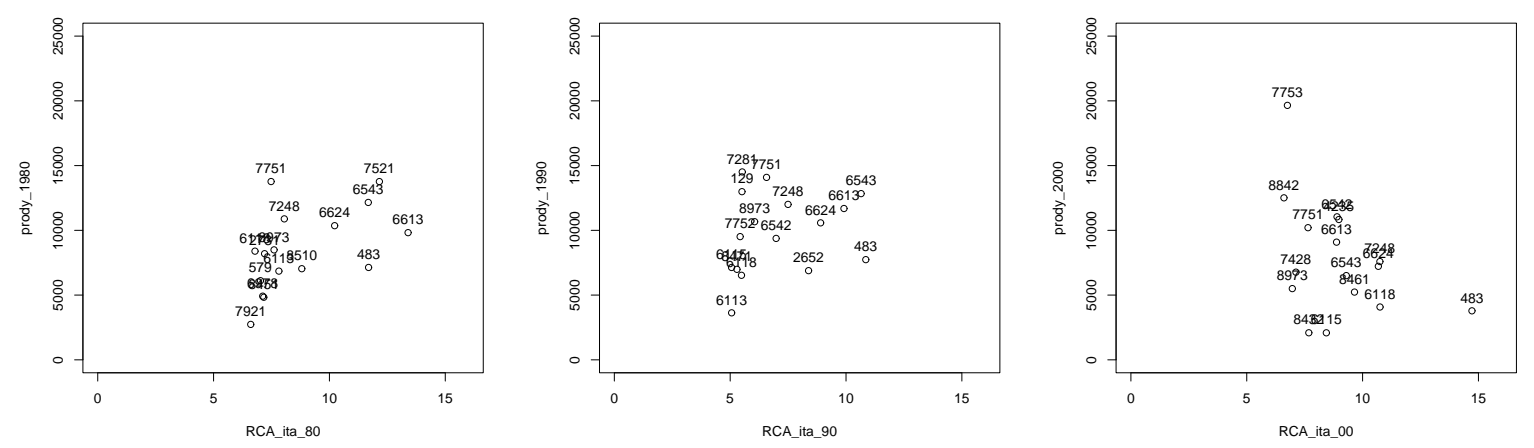

strong and persistent presence in sectors characterized by decreasing 'sophistication'. This is particularly apparent for sectors 6115 (Sheep and Lamb Skin Leather) and 8432 (Suits and Costumes for womens, of Textile fabrics), wherein the RCA has more than doubled, while the PRODY index in 2000 is less than half it was in 1980. Less pronounced, but still there, the same pattern occurs also in sector 483 (Pasta and Similar Products), where the Italian RCA passed from 11.7 in 1980 to 14.7 in 2000, while the PRODY level associated with this sector has decreased from 7133 US Dollars to less than 3784 US Dollars. Something similar happened with two other sectors in which Italy traditionally displays high level of specialization, that is sectors 6118 (Leather, specially dressed or finished) and 6624 (Non-refractory Ceramic Bricks, Tiles, Pipes and Similar Products). And the same is actually true for sector 7751(Household Type Laundry Equip.) which decreases its PRODY from around 13768 to less than 10204 US Dollars. Obviously, there are also cases wherein Italy has increased its specialization level in sectors recording increases in the PRODY index, but the overall picture confirms the negative association between the sectoral income/productivity content and the extent of specialization suggested above as a peculiar feature characterising the Italian case in the last decade. The relevance of the finding seems particularly important also in view of the fact that these sectors are neither outliers nor marginal in the structure of the Italian export. We have marked with an asterisk those whose share in overall exports was, in each year, below the average sectoral share: only 2 out of 15 remain constantly below average during the period, while the others, even if relatively small before 2000 , all have become relatively large by that time.

\section{Conclusion and further research}

This paper provided an empirical assessment of the 'sophistication'of the Italian specialization pattern and of its evolution during the last 25 years. We began exploring the inter-temporal dynamics of the PRODY index, a new index measuring sector's 'sophistication' recently proposed in Hausmann et al. (2005). A first contribution of our paper was precisely to highlight some of the properties of this index and to gather novel empirical evidence on its evolution over time. In particular, extending the work by Hausmann et al. (2005), we have calculated the index 
Table 7: Top 15 Italian RCA and the associated PRODY, dynamics

\begin{tabular}{r|rc|rc|rc}
\hline \hline & \multicolumn{2}{|c}{1980} & \multicolumn{2}{c}{$\mathbf{1 9 9 0}$} & \multicolumn{2}{c}{$\mathbf{2 0 0 0}$} \\
Sector & PRODY & RCA & PRODY & RCA & PRODY & RCA \\
\hline \hline 483 & 7133 & 11.7 & 7737 & 10.9 & 3784 & 14.7 \\
6118 & 8393 & $6.8^{*}$ & 6529 & $5.5^{*}$ & 4079 & $10.8^{*}$ \\
7248 & 10885 & 8.1 & 12004 & 7.5 & 7590 & 10.7 \\
6624 & 10359 & 10.2 & 10579 & 8.9 & 7222 & 10.7 \\
8461 & 8177 & $6.2^{*}$ & 3321 & $0.0^{*}$ & 5232 & $9.7^{*}$ \\
6543 & 12154 & 11.7 & 12835 & 10.7 & 6505 & 9.3 \\
4235 & 6516 & $2.8^{*}$ & 9046 & 4.2 & 10836 & 9.0 \\
6542 & 9594 & 4.5 & 9376 & 7.0 & 11044 & 8.9 \\
6613 & 9822 & 13.4 & 11685 & 9.9 & 9088 & 8.9 \\
6115 & 5925 & $2.1^{*}$ & 7132 & 5.1 & 2086 & 8.4 \\
8432 & 5086 & $3.0^{*}$ & 5713 & $1.8^{*}$ & 2089 & 7.7 \\
7751 & 13768 & 7.5 & 14088 & 6.6 & 10204 & 7.6 \\
7428 & 12234 & 3.5 & 11314 & 3.8 & 6770 & 7.1 \\
8973 & 8491 & 7.6 & 10666 & 6.1 & 5500 & 7.0 \\
7753 & 15818 & $4.1^{*}$ & 16858 & $3.6^{*}$ & 19651 & 6.8 \\
8842 & 9958 & 3.1 & 7250 & 3.7 & 12510 & 6.6 \\
\hline \hline
\end{tabular}

PRODY is measured in 1996 PPP-adjusted US dollars per capita. * The export share of the sector is below the average sectoral export share in that year.

for 1980, 1990 and 2000, and mapped the dynamics through these reference years. We found that the characteristics of a virtuous specialization pattern have changed with time and that, in some cases, what was worth exporting two decades ago it is less so now. This implies that each country, in order to gain from trade (and spur growth), is required to continually adapt to this changing environment.

Second, we have proposed to look at the co-evolution of the PRODY index and the $R C A$ index in order to evaluate whether the specialization pattern of a country is well directed or not. We have applied this methodology to the analysis of the Italian 'anomaly'. On the one hand, we found that, consistently with previous studies, the Italian specialization pattern has been highly persistent over time. On the other hand, the evolution of the PRODY index in those sectors where Italy has been, and still is, highly specialized suggests that the contribution of export to growth has become weaker and weaker. Indeed, Italy is competitive and specialized in products that are loosing importance in terms of income/productivity content in world trade. Thus, while high persistence of the specialization pattern it is not a problem per se, our analysis shows that the worry about the Italian anomaly is justified by the fact that, while world trade is rapidly evolving, Italy is stuck with its old model which is now becoming wrong. 
The present analysis could be extended in different directions. We sketch here some of them. In the present paper we computed RCA, and, consequently, the PRODY indeces, using overall world trade flows. But one could also consider some other meaningful benchmark, as OECD countries, or split the analysis into two parts, distinguishing, for instance, developed vs low-medium income countries in order to evaluate the evolution of the country's specialization pattern. In the same spirit, one could also play with the sample of sectors. While an high level of disaggregation is important for many purposes, "aggregating " back the data could also offer a way to better grasp whether there are patterns common to products that are similar with respect to, for instance, technological content (think about Pavitt taxonomy) or other factors. Finally, it would interesting to replicate the analysis with some countries other than Italy. Such an attempt, together with providing a sort of test of how much informative is this methodology, would also offer a basis for useful comparisons among the patterns experienced by different countries.

All these developments are left for future work.

\section{References}

Brasili, A., Helg, R. and P. Epifani, 2000. On the dynamics of trade patterns. De Economist, vol. $148(2), 233-257$

Epifani, P., 1999. Sulle determinanti del modello di specializzazione internazionale dell'Italia. Politica Economica, 15 (2), 195-224

De Benedictis, L., 2006. Three decades of Italian comparative advantages. The World Economy, forthcoming

De Benedictis, L. and Tamberi, M., 2004. Overall specialization empirics: techniques and applications. Open Economies Review, vol. 15, 323-346

De Benedictis, L., Tamberi, M. and M. Gallegati, 2006. Semiparametric analysis of the specialization-income relationship. Applied Economic Letters, forthcoming

Dalum, B., K. Laursen and B. Verspagen, 1999. Does specialization matter for growth? Industrial and Corporate Change, vol 8(2), pp. 267-288

Dosi, G., Pavitt K. and L. Soete, 1990. The Economics of Technical Change and International Trade. Harvester Wheatsheaf, New York 1990

Durlauf, S.N. and Quah, D.T., 1999. The new empirics of economic growth, in J. Taylor and M. Woodford (eds.), Handbook of Macroeconomics, North-Holland

Faini, R., 2004. Fu vero declino? L'Italia negli anni '90, Il Mulino, pp. 1072-1083

Feenstra, R.C. and Rose, A. K., 2000. Putting things in order: patterns of trade dynamics and growth. The Review of Economics and Statistics, vol. 82(3), 369-382 
Grossman, G.M. and Helpman, E., 1991. Innovation and Growth in the Global Economy. MIT Press, Cambridge

Hausmann, R., Hwang, J. and D. Rodrik, 2005. What you export matters. NBER Working Paper, No. 11905

Huber, P.J., 1967. The behavior of maximum likelihood estimates under nonstandard conditions. Proceedings of the Fifth Berkeley Mathematical Symposium on Mathematical Statistics and Probability, Vol. 1, 221-223

Kaldor, N., 1966. Causes of the slow rate of growth of the United Kingdom. Cambridge University Press, Cambridge

Lall, S., Weiss, J. and J. Zhang, 2006. The sophistication of exports: a new measure of product characteristics. QEH Working Paper Series, No. 123

Monti, P., 2005. Caratteristiche e mutamenti della specializzazione delle esportazioni italiane, Temi di Discussione n. 559, Banca dItalia

Michaely, M., 1984. Trade, income levels, and dependence. North-Holland, Amsterdam

Onida, F., 1999. Quali prospettive per il modello di specializzazione internazionale dell'Italia. Economia Italiana, vol. 3, 573-626

Pasinetti, L. L., 1981. Structural change and economic growth. Cambridge University Press, Cambridge

Proudman, J. and Redding, S., 2000. Evolving patterns of international trade. Review of International Economics, vol. 8 (3), 373-396

Quah, D.T., 1996. Empirics for economic growth and convergence. European Economic Review, vol.90, pp. $1353-1375$

Redding, S., 1999. Dynamic comparative advantage and the welfare effects of trade. Oxford Economic Papers, vol. 51, pp. 1539.

Rodrik, D., 2006. What's so special about China's exports? China $\&$ World Economy, forthcoming

Silverman, B.W., 1981. Using kernel density estimates to investigate multimodality. Journal of the Royal Statistical Society, Ser. B 43, 97-99

Silverman, B W., 1983. Some properties of a test for multimodality based on kernel density estimates, in Probability, Statistics and Analysis, J. F. C. Kingman and G. E. H. Reuter (eds.), 248-259. Cambridge University Press, Cambridge, UK

Silverman, B.W., 1986. Density estimation for statistics and data analysis. Monograph on statistics and applied probability, No. 26, Chapman and Hall, London 
Thirwall, A.P., 1979. The balance of payments constraint as an explanation of international growth rate differences. Banca Nazionale del Lavoro Quarterly Review, vol. 3, 245-53 


\section{Appendix}

\section{Proof of Proposition 3}

We now derive the effect of changes in the number of exporting countries on the values of the $P R O D Y$ index in sector $l$. Consider the following situation where at time $t$ two countries, $A$ and $B$, are exporting in sector $l$ and a third country $C$ enters that market in $t+1$. Suppose that incumbents' exports in all the sectors ( $l$ included) remain constant, and that the same happens with their (real) per-capita incomes. Under these conditions, sector $l$ 's share in world exports will increase, causing a decrease in the $R C A_{l}$ of both country $A$ and $B\left(\Delta R C A_{i, l}<0\right)$, and an obvious increase from zero to a positive number in the RCA of the entrant country $C$ $\left(\triangle R C A_{C, l}>0\right)$. Now define

$$
\Delta S=\sum_{i}\left(R C A_{i, l}\right)_{t+1}-\sum_{i}\left(R C A_{i, l}\right)_{t}
$$

and suppose that country $C$ does enter with an $R C A_{l}$ relatively high with respect to the incumbents, so that

$$
R C A_{C, l}>\left|\Delta R C A_{A, l}\right|+\left|\Delta R C A_{B, l}\right|
$$

and, thereby, $\Delta S>0$. Ask now the question about under which circumstances the entry of country $C$ will cause an increase in $P R O D Y_{l}$. With some simple algebra one has that the inequality

$$
\Delta P R O D Y_{l}=\frac{\Delta R C A_{A, l}}{\Delta S} \cdot y_{A}+\frac{\Delta R C A_{B, l}}{\Delta S} \cdot y_{B}+\frac{R C A_{C, l}}{\Delta S} \cdot y_{C}>0
$$

is satisfied, given condition (11), whenever

$$
y_{C}>-\frac{\Delta R C A_{A, l}}{R C A_{C, l}} \cdot y_{A}-\frac{\Delta R C A_{B, l}}{R C A_{C, l}} \cdot y_{B}
$$

or, alternatively, when

$$
R C A_{C, l}>\underbrace{-\Delta R C A_{A, l}}_{>0} \cdot \frac{y_{A}}{y_{C}} \underbrace{-\Delta R C A_{B, l}}_{>0} \cdot \frac{y_{B}}{y_{C}}
$$

In words, the effect of a new country starting exporting in sector $l$ on the PRODY index crucially depends on the income level of the countries involved: both condition (13) and condition (14) show that the probability of observing an inter-temporal increase in $P R O D Y_{l}$ is higher the more developed the entrant is, and the less developed the already exporting countries are.

\section{Data description}

Our trade data come from the UN Commodity Trade Statistics Database (COMTRADE). For each country present in the dataset for the period under consideration, it contains detailed yearly figures on the value of exports in 777 sectors (SITC Rev.2 classification, 4-digit) and aggregate data on the economy wide level of exports. To compute the Balassa-RCA index we also need, for each year, the time series about the value of world exports in each single sector and about the overall value of world exports. Both the series are also reported in COMTRADE. The major 
caveat concerns the number of countries included, which varies from year to year, for reasons going from simple non-reporting to processes of integration and disintegration, or changes of name, occurred in the period considered.

The same happens also with the Penn World Tables, from which we get the data about real per-capita GDP's. This fact represents a potential drawback since, as mentioned in the text, a careful construction of the PRODY index requires to follow an as large as possible and balanced panel of countries over the whole time span, so that obversed changes in the index result from actual trade and income dynamicsa, rather than simple lost of data in a particular year.

To cope with that, a first choice we made was to restrict the attention to three years (1980, 1990 and 2000), as reference for inter-temporal comparisons. Then, one would be tempted to consider only those countries for which both trade and income data are available in these years, but one can actually do something better in order to fully exploit the trade data. To understand why, why this procedure just recall the definition of the index given in the text (see equation 1 above) and note that the weights assigned to each country per-capita GDP's, $s_{i, l}$, are computed considering all the countries exporting good $l$ in each year. Take, for instance, the hypothetical situation where only two countries, say the US and Bangladesh, export in sector $l$, and imagine to have income data for all the years only for the US. Note that this is likely to be the typical situation, since non-reporting rates, actually in both trade and income data, are usually much higher among low-medium income countries, especially at the beginning of the time period. Further, suppose that Bangladesh, given its relatively un-differentiated export bundle, is highly specialized in export of commodity $l$, while the US have only a moderate $R C A_{l}$, so that $s_{i, l}$ is very high for Bangladesh (say 0.9) and very low (0.1) for the US. Now, excluding Bangladesh tout court from the sample would assign a weight $s_{i, l}=1$ to the US, resulting in a value of $P R O D Y_{l}$ artificially high and equal to US per-capita income. We proceeded in two steps. First, we computed the $s_{i, l}$ using all the countries present in the COMTRADE dataset in each single reference year, no matter whether they were also included in the Penn World Tables, nor whether they were present in all the three years 15 Only after that we worried about which sample of country to include in the computation of the sectoral PRODY indeces. Obviously, the most reasonable choice to keep inter-temporal comparability under control as much as possible was simply to use only the data relative to those countries for which figures on per-capita GDP were available in all of the three reference years. Whenever possible, we tried to "save" as many countries as we can, keeping track, in particular, of changes in the name and unification or disintegration processes: in the end, we were left with 90 countries. 16

\footnotetext{
${ }^{15}$ Of course not all the countries do export in all of the 777 sectors, and when a zero is reported one has simply to assume that it is precisely because that particular country is not exporting in that particular sector.

${ }^{16}$ For instance, COMTRADE reports exports data for Benelux in 1980, but for Belgium and Luxembourg, separately, in 1990 and 2000. Our choice was to keep the two countries together into a fictitious aggregate entity, attributing to it the sum of the two countries' per-capita GDP's, divided by two. The same we did also with Slovak Republic and Czech Republic, reported as Czechoslovakia in 1980, before they split.
} 


\section{The dynamics of the PRODY index: a tale of four sectors}

To offer additional understandings on how the PRODY indeces evolved over time, we proceed with a very simple investigation. In Table 8 we report the values of the PRODY index for the 20 best ranked sectors in each of the three reference years, and we track back which are the countries involved in world exports of these products in each year, and the evolution of their associated $s_{i, l}$, the normalized $R C A$ appearing in the definition of PRODY. This shows many interesting examples of the various dynamics present in the data, that we summarize focusing on 4 of them.

If we closely look at Sector 6733 (Angles, Shapes, Sections and Sheet Pilling of Iron or Steel), ranked first in 2000, we see that its high PRODY value is mainly due to the fact that Luxembourg, a country with one of the highest per capita income in 2000, exhibits a normalized RCA in this sector of approximately 0.45 .17

An instructive picture emerges when one focuses on sector 121 (Bacon, Ham and other Dried, Salted, Smoked Meat). In all of the three years considered, Denmark is the most specialized country, with a normalized RCA of about 0.45 in 2000 and 0.5 in the previous periods, while Italy, Luxembourg and The Netherlands represent substantially all the remaining part, with $s_{i, l}$ varying between 0.1 and 0.2 . Thus, it comes with not much surprise that the ranking in terms of PRODY follows the increase in the income of Denmark and of the other main players: out of the top 20 in 1980 ( $P R O D Y \simeq 12000$ US Dollars, not reported in the Table), the sector moves to the tenth place in 1990 and is second in 2000). We have here a prototypical example of a sector where the PRODY index moves together with the GDP of the major exporting countries, and ranks very well exactly because the main exporters are rich countries.

The dynamics of Sector 6812 (Platinum and Other Metals of the Platinum), third in 2000, are even more instructive. Though UK is also important (with $s_{i, l} \simeq 0.2$ ), Switzerland is the main responsible for the high ranking in 2000, as its normalized RCA is approximately 0.6 and it has one of the highest GDP per capita in this year. The sector was well positioned (12th) also in 1980, when very few countries, and mainly rich (Switzerland and UK, again) were significantly specialized in the export of this industry. On the contrary, the value of the index falls dramatically to approximately 9500 US Dollars in 1990, around the 400th place in

\footnotetext{
17 Note that sector 6733 was not in the top 20, neither in 1990, nor in 1980, apparently in contrast with one might expect, given the observed stable ranking. The reason for this is particularly interesting, as it shows how sensitive the index is with respect to data constrains. Indeed what happens here is simply that, as mentioned in Section 6, for 1980 and 1990 we only have export data about Benelux, rather that about Belgium and Luxembourg separately and we built a fictitious "aggregate country" attributed to Benelux the sum of per capita GDP's of Belgium and Luxembourg, divided by two. Thus, the extent of specialization in this sector in 1980 and 1990 , presumably very high for Luxembourg alone, is mitigated by the low specialization of Belgium, so that the normalized RCA computed for Benelux as a whole is much lower than that for Luxembourg alone. The same happens with per capita incomes: aggregating the two countries fictitiously lowers the income of the -presumablymost specialized actor in this sector, Luxembourg. Both the effects are clearly likely to be responsible for the disappearance of sector 6733 from the list in 1980 and 1990: haven't we had to cope with these data driven problems, 6733 would have probably had a high index also before 2000, confirming the overall picture of relative stability.
} 
Table 8: Top-20 PRODY sectors, dynamics

\begin{tabular}{cc|cc|cc}
\hline \hline \multicolumn{2}{c}{1980} & \multicolumn{2}{c}{1990} & \multicolumn{2}{c}{ 1900 } \\
Sector & PRODY & Sector & PRODY & Sector & PRODY \\
\hline \hline 3415 & 20,740 & 451 & 20,571 & 6733 & 24,420 \\
6880 & 19,580 & 7268 & 20,320 & 121 & 24,329 \\
7268 & 19,488 & 9510 & 20,048 & 6812 & 22,886 \\
7187 & 18,406 & 2860 & 19,394 & 6880 & 22,879 \\
2226 & 18,188 & 8996 & 19,313 & 6631 & 22,080 \\
7245 & 17,917 & 7741 & 19,302 & 5147 & 22,069 \\
5147 & 17,885 & 2120 & 19,147 & 5156 & 21,835 \\
7129 & 17,811 & 7251 & 19,099 & 6647 & 21,557 \\
7126 & 17,366 & 8744 & 19,011 & 8813 & 21,130 \\
7911 & 17,266 & 121 & 18,981 & 7251 & 20,544 \\
6812 & 17,239 & 6642 & 18,937 & 5332 & 20,364 \\
7252 & 16,884 & 7259 & 18,875 & 6644 & 19,977 \\
8996 & 16,753 & 7252 & 18,844 & 7268 & 19,962 \\
5163 & 16,739 & 7423 & 18,671 & 5827 & 19,797 \\
7264 & 16,587 & 5827 & 18,670 & 7753 & 19,651 \\
7412 & 16,556 & 6411 & 18,635 & 6572 & 19,609 \\
7434 & 16,545 & 8851 & 18,556 & 6412 & 19,560 \\
5411 & 16,535 & 5411 & 18,457 & 7213 & 19,479 \\
7784 & 16,521 & 7931 & 18,402 & 7412 & 19,387 \\
7246 & 16,473 & 7264 & 18,376 & 6418 & 19,160 \\
\hline \hline & \multirow{2}{*}{ Values in 1996 PPP-adjusted US dollars } &
\end{tabular}

that year. The reason for this fact is easily recovered by looking at exports figures. In 1990 many countries enter the market, and they are all countries with low-medium level of per-capita income. Accordingly, though the extent of specialization of the rich countries active in the sector, Switzerland and UK, remains high and similar to that of 1980, the weights $s_{i, l}$ assigned to their per-capita incomes in the computation of the index are much lower. What happens with Switzerland is particularly striking, with $s_{i, l} \simeq 0.04$ in 1990 . We observe here what we suggested has likely occurred over time in most of the industries: consistently with Figure 1, the entry of LDC countries during the '80s has likely produced a widespread reduction in the values of $P R O D Y$. Then, when rich countries take again the "leadership", or at least a prominent role in terms of relative weigh, as it is the case for Switzerland in 2000, the index becomes high again, while, on the contrary, if low-medium income countries keep a strong presence, the index further falls. This is confirmed, for instance, by looking at the dynamics in Sector 3415 (Coal Gas, Water Gas, Producer Gas and Similar Gases), out of the top 20 in 2000, but the best 
ranked in 1980. In this year Switzerland, again, has a very high normalized RCA, but then its weigh becomes almost zero as time goes by, and in 2000 we end up with low income countries (Zimbabwe, Chad and Qatar) having the highest shares. 